\title{
Influência do treinamento concor- rente na composição corporal e óssea de adolescentes obesos
}

\section{Influence of concurrent training on body and bone composition of obese adolescents}

\author{
Paula A Monteiro ${ }^{1}$, Barbara de M M Antunes ${ }^{1}$, Loreana S Silveira ${ }^{1}$, Ricardo R Agostinete ${ }^{3}$, \\ Malena R Picolo ${ }^{2}$, Ismael F Freitas Junior ${ }^{3}$
}

\begin{abstract}
RESUMO
Modelo de estudo: Estudo prospectivo

Objetivo: Analisar a diferença da densidade, conteúdo mineral ósseo e composição corporal de adolescentes obesos pré e pós treinamento concorrente de 16 semanas. Métodos: Amostra composta por 19 adolescentes obesos. Mensurou-se a densidade mineral óssea(BMD), conteúdo mineral ósseo(BMC), massa corporal magra(MCM) em kg, massa de gordura(MG) em kg e em percentual(\%GC) e percentual de gordura do tronco(\%GT) por meio da técnica absortiometria de raios-X de dupla energia(DEXA). A Intervenção consistiu de treinamento concorrente (treino resistido [30 minutos] e aeróbio [30 minutos]) com três sessões semanais. Para análise estatística utilizou-se teste t de Student, qui-quadrado, ANOVA e correlação de Pearson por meio do software SPSS(17.0) e significância estatística fixada em $p<5 \%$. Resultados: Houve diferença estatística entre os gêneros para \% $\mathrm{GT}(\mathrm{p}<0,007)$. No grupo feminino houve correlação entre BMD, \%GC e \%GT, já a BMC correlacionou-se com peso e MCM. No grupo masculino houve correlação da BMC e BMD com a estatura e MCM. Após a intervenção, no grupo feminino, houve significância apenas para estatura $(p<0,014)$, já no grupo masculino houve aumento significativo da estatura $(p<0,000), \operatorname{MCM}(p<0,011)$ e BMC $(p<0,002)$ bem como a diminuição das variáveis $\operatorname{IMC}(p<0,004)$, $\% G C(p<0,000)$ e $\% G T(p<0,016)$. Após o treinamento $78,9 \%$ dos adolescentes aumentaram e $21,1 \%$ diminuíram o BMD. Dos que aumentaram o BMD 63,2\% diminuíram o \%GC, 57,9\% a MG e $57,9 \%$ a MCM. Conclusão: Após a intervenção houve diminuição da gordura corporal, aumento da massa magra e conteúdo mineral ósseo de adolescentes do sexo masculino. No grupo feminino não houve diferença entre os momentos.
\end{abstract}

Palavras Chave: Exercício. Densidade Óssea. Obesidade.

1. Departamento de Educação Física - Universidade Estadual Paulista, Campus de Rio Claro, Brasil-SP.

2. Departamento de Fisioterapia - Universidade Estadual Paulista, Campus de Presidente Prudente, Brasil-SP.

3. Departamento de Educação Física - Universidade Estadual Paulista, Campus de Presidente Prudente, Brasil-SP.
Correspondencia

Paula Alves Monteiro

UNESP, Departamento de Educação Física. Rua Roberto Simonsen no 305, Vila Santa Helena, CEP: 19060-900 / Presidente Prudente/SP.

Artigo recebido em 03/01/2014 Aprovado para publicação em 10/10/2014 


\begin{abstract}
Study Design: Prospective study

Objective: Analyze the difference of density, bone mineral content and body composition of obese adolescents before and after training concurrent of 16 weeks. Methods: The sample consisted of 19 obese adolescents. We measured bone mineral density(BMD), bone mineral content(BMC), lean body mass(MCM), fat mass(MG) in $\mathrm{kg}$ and percentage(\%BF) and percentage trunk fat(\%GT) by the technique of X-ray absorptiometry dual energy absorptiometry(DEXA). The intervention consisted of concurrent training (resistance training [30 minutes] and aerobic [30 minutes]) with three weekly sessions. Statistical analysis was made by Student's t test, chi-square, ANOVA and Pearson correlation using SPSS software(17.0), and statistical significance set at $p<5 \%$. Results: There were significant differences between the gender for the GT\%(p<0.007). In the female group correlation of BMD with BF\% and \%GT, since the BMC correlated with weight and MCM. In the male group there was correlation of BMC and BMD with height and MCM. After the intervention, the female group was significant only for height $(p<0.014)$, as in the male group there was an increase in height $(p<0.000), \operatorname{LBM}(p<0.011)$ and BMC $(p<0.002)$ was significantly and as the decrease in $\mathrm{BMI}(\mathrm{p}<0.004), \% \mathrm{BF}(\mathrm{p}<0.000)$ and $\mathrm{GT} \%(\mathrm{p}<0.016)$. After training, $78.9 \%$ of adolescents increased the BMD and $21.1 \%$ decreased. That the increased BMD decreased $63.2 \%$ BF\%, $57.9 \%$ and 57.9\% MG and MCM. Conclusion: After the intervention, there was a decrease body fat, an increase in lean body mass and bone mineral content in male adolescents. In the female group there was no difference from pre to post intervention.
\end{abstract}

Keywords: Exercise, Bone Density. Obesity.

\section{Introdução}

A prevalência de obesidade na população pediátrica é observada de forma alarmante em todas as faixas etárias e em ambos os gêneros em diversos países, independentemente das condições de desenvolvimento, tornando-se uma epidemia mundial e um grave problema de saúde pública. ${ }^{1}$

De acordo com a Pesquisa de Orçamento Familiar 2008-2009, realizada pelo Instituto Brasileiro de Geografia e Estatística (IBGE), a quantidade de pessoas com excesso de peso no Brasil aumentou substancialmente em todas as faixas etárias, sendo que o sobrepeso atinge $30 \%$ das crianças com idade entre cinco e nove anos de idade, aproximadamente $20 \%$ da população entre 10 e 19 anos, e $48 \%$ das mulheres e 50,1\% dos homens acima de 20 anos. $^{2}$

A obesidade é caracterizada como uma doença de origem multifatorial, decorrente de fatores endógenos e exógenos, e que associa-se positivamente com um estado inflamatório podendo desencadear diversas alterações metabólicas tais como dislipidemias, diabetes tipo 2, hipertensão arterial, alterações no metabolismo da glicose, esteatose hepática, alterações cardiorrespiratórias, cânceres, entre outras doenças. ${ }^{3}$

Estudos têm mostrado que crianças obesas possuem maior conteúdo e densidade mineral óssea, e que o desenvolvimento ósseo pode ser influenciado por diversos fatores, dentre eles a massa magra e a gordura corporal, no entanto essa relação sobre a saúde óssea de obesos ainda não esta bem definida. ${ }^{4}$

Para minimizar o crescimento da obesidade bem como das alterações metabólicas e ortopédicas associadas faz-se necessária à adoção de medidas preventivas e de tratamento desta doença, sugerindo a associação entre exercício físico e reeducação alimentar. ${ }^{5}$ Estudos apresentam a potencialização dos resultados benéficos quando realiza-se uma intervenção multidisciplinar por meio de modificações na dieta, implementação de atividade física sistematizada e acompanhamento médico como observado no estudo de Fagherazzi et al. ${ }^{6}$

Dentre os diferentes modelos de exercício físico, o treinamento concorrente (TC), composto de exercício aeróbio e resistido em uma mesma sessão de treinamento $^{7}$, pode ser indicado para a manutenção da massa óssea, pois ambos os modelos são apontados como atividades que resultam em osteogênese. $\mathrm{O}$ mecanismo pelo qual esses processos ocorrem, é pela magnitude da deformação óssea ocorrida durante essas atividades através das contrações musculares, onde maiores cargas e intensidades de treino resultam em respostas maiores da massa óssea. ${ }^{8}$

Diante disto o objetivo do presente estudo é analisar a diferença da densidade, conteúdo mineral ósseo e composição corporal de adolescentes obesos pré e após treinamento concorrente de 16 semanas. 


\section{Materiais e Métodos}

O presente estudo de caráter longitudinal foi composto por participantes do projeto de extensão universitária, denominado "Super Ação" que oferece a população de Presidente Prudente e região tratamento não farmacológico e multidisciplinar à obesidade infanto-juvenil, sendo o mesmo desenvolvido no Departamento de Educação Física da Universidade Estadual Paulista-UNESP, Campus de Presidente Prudente. Para ingresso no referido programa adotaramse os seguintes critérios de inclusão: (a) o jovem ser classificado obeso pelo Índice de Massa Corporal (IMC), seguindo critério publicado por Cole et $\mathrm{al}^{9}$.; (b) ter entre 12 e 17 anos completos, na data da avaliação; (c) não apresentar nenhum problema de ordem clínica que impossibilite a prática de atividades físicas e; (d) os pais ou responsáveis legais assinarem o termo de consentimento formal para participação no programa. Os participantes que atingiram três faltas consecutivas ou aleatórias durante a intervenção foram excluídos do referente programa de extensão.

Foram avaliados trinta e nove jovens, vinte deles foram excluídos da amostra por excesso de faltas (presença mínima de 95\% no programa). Desta forma, para a presente pesquisa fizeram parte da amostra 19 adolescentes obesos, participantes do programa para tratamento da obesidade. Todos os participantes passaram por avaliação da composição corporal antes de iniciar as atividades e ao final do estudo as avaliações foram realizadas novamente.

Do ponto de vista ético, todos os cuidados foram tomados. Cada sujeito foi convidado a participar, voluntariamente, do estudo e, juntamente com seus pais ou responsáveis legais, informados detalhadamente sobre objetivos e, também, como os dados seriam coletados. Somente participaram aqueles que apresentaram o termo de consentimento formal e esclarecido, devidamente assinado. $\mathrm{O}$ presente estudo foi aprovado pelo Comitê de Ética em Pesquisa da FCT/UNESP com número de protocolo 07/2009.

\section{Triagem e avaliações iniciais}

A primeira triagem foi composta de mensuração da massa corporal, aferida com a utilização de uma balança eletrônica da marca Filizola, com precisão de $0,1 \mathrm{~kg}$ e capacidade máxima de $180 \mathrm{~kg}$, de acordo com a metodologia proposta por Freitas Jr et al. ${ }^{10}$ e pela estatura, que foi aferida com a utilização de um estadiômetro fixo, marca Sanny, com precisão de
0,1 cm e extensão máxima de dois metros, de acordo com a metodologia proposta por Freitas Jr et al. ${ }^{10}$ Por meio destas medições foi calculado o IMC. Em seguida os adolescentes foram submetidos a avaliações da composição corporal pela densidade óssea. Todas as avaliações foram repetidas ao final do estudo.

\section{Avaliação da composição corporal}

Para a análise da composição corporal foi empregada à técnica da absortiometria de raios-X de dupla energia (DEXA), utilizando-se o equipamento modelo GE Lunar - DPX-NT e software 4.7. Desta forma foram quantificadas as variáveis: densidade mineral óssea (BMD), conteúdo mineral ósseo (BMC), massa Corporal Magra (MCM) em kg, massa de gordura (MG) em kg e em percentual (\%GC) e percentual de gordura do tronco (\%GT).

\section{Treinamento Concorrente}

Durante 16 semanas, o programa de intervenção foi fundamentado no treinamento concorrente (TC), composto de exercício aeróbio e resistido em uma mesma sessão de treino ${ }^{7}$, com frequência semanal de três vezes e duração de 60 minutos por sessão (aproximadamente 50\% do tempo do treino aeróbio e $50 \%$ do tempo do treino resistido (musculação). ${ }^{11}$ Foram realizados exercícios de alongamento no início e final do treino e os participantes foram orientados a ingerir água e trajar roupas adequadas para a prática de exercícios físicos.

Para determinação da intensidade do treino aeróbio, os adolescentes realizaram um teste de esforço máximo progressivo e contínuo em esteira ergométrica modelo ATL (marca Inbrasport, BR), para usuários de até $180 \mathrm{~kg}$, com 0 a $26 \%$ de inclinação e velocidade de até $24 \mathrm{~km} / \mathrm{h}$. O teste de esforço iniciouse a uma velocidade de $3 \mathrm{~km} / \mathrm{h}$, durante um minuto a uma inclinação de $1 \%$. A cada minuto houve um incremento de $0,5 \mathrm{~km} / \mathrm{h}$ na velocidade mantendo-se a inclinação em $1 \%$, respeitando um período máximo de 12 minutos de teste. ${ }^{12}$ Durante todo o teste de esforço os avaliados permaneceram com uma máscara de silicone com pneumotacografo de médio fluxo (10 até 120 l/min). Por meio do teste de esforço estabeleceu-se o VO2 pico e a frequência cardíaca para o controle da intensidade do treinamento aeróbio.

O treino aeróbio consistiu de caminhada e corrida em uma pista de atletismo oficial de $400 \mathrm{~m}$ de extensão. A intensidade do esforço foi monitorada por meio do monitor de frequência cardíaca (marca Po- 
lar ${ }^{\circledR} \mathrm{S} 810$ ) colocado, aleatoriamente, em quatro indivíduos a cada sessão. O equipamento foi ajustado para que se tenha garantia de que os participantes permanecessem na zona aeróbia de treino (1-4 semana: 65\% $\mathrm{VO}_{2}$ pico; 5-8 semana: 70\% $\mathrm{VO}_{2}$ pico; 9-12 semana: $80 \% \mathrm{VO}_{2}$ pico; $13-16$ semana: $85 \% \mathrm{VO}_{2}$ pico), para isso foi utilizada a frequência cardíaca encontrada na mesma intensidade durante a realização do teste de esforço máximo.

Para a determinação da intensidade do treinamento resistido, após o período de quatro semanas de adaptação neuromuscular, utilizando-se de cargas mínimas e uma única série, os avaliados foram submetidos ao teste de predição de uma carga máxima por meio do teste de 10 repetições máximas (10RM), segundo Baechle ${ }^{13}$, com uma carga máxima individual para a realização de 10 repetições em cada exercício. Para o teste de 10 RM realizou-se os exercícios Leg Press $45^{\circ}$, Supino reto, Remada baixa e Rosca direta, a fim de analisar grandes grupos musculares.

Após o período de adaptação o treino iniciouse com intensidade de $45 \%$ do RM e acrescido de 5\% a cada duas semanas, totalizando uma intensidade final de $80 \%$ do RM. No segundo e terceiro mês, o treino foi realizado com uma serie de 20 a 25 repetições e no quarto e quinto mês, o treino será realizado com uma serie de 8 a 15 repetições para cada exercício.
O treino resistido foi realizado em forma de circuito na academia de musculação.

\section{Análise estatística}

Foi calculada média, como medida de tendência central e desvio-padrão e intervalo de confiança de $95 \%$. Para descrição da amostra, bem como a comparação entre grupos, masculino e feminino, foi realizado o teste ANOVA. O teste $t$ de Student pareado foi empregado para verificar a existência de diferenças estatísticas entre as variáveis em ambos os momentos de estudo e a correlação de Pearson para analisar a associação entre as variáveis. O teste qui-quadrado foi realizado para descrever a frequência dos adolescentes que aumentaram ou diminuíram a BMD de acordo com o comportamento da composição corporal pós intervenção.

O software estatístico SPSS (versão 17.0) foi utilizado em todas as análises e foram considerados estatisticamente relevantes, apenas valores de significância (p) inferiores a 5\%.

\section{Resultados}

A descrição da amostra separada por gênero e a correlação da densidade e conteúdo mineral ósseo com as variáveis de composição corporal (Tabela 1),

Tabela 1. Descrição da amostra separada por gênero e correlação da densidade e conteúdo mineral ósseo com as variáveis de composição corporal.

\begin{tabular}{|c|c|c|c|c|c|}
\hline & $\begin{array}{r}\text { Feminina } \\
\text { Media }\end{array}$ & $\begin{array}{l}(n=6) \\
(D P)\end{array}$ & $\begin{array}{r}\text { Masculina } \\
\text { Media }\end{array}$ & $\begin{array}{l}(n=13) \\
(D P)\end{array}$ & $p$ \\
\hline Idade & 13,8 & $(0,7)$ & 13,5 & $(1,4)$ & 0,648 \\
\hline Peso(kg) & 83,8 & $(13,0)^{b}$ & 88,3 & $(15,8)$ & 0,555 \\
\hline Estatura(cm) & 158,7 & $(11,6)$ & 165,9 & $(6,6)^{a b}$ & 0,097 \\
\hline IMC & 33,1 & $(1,7)$ & 32,0 & $(5,7)$ & 0,686 \\
\hline$\% G C$ & 49,2 & $(4,6)^{a}$ & 44,3 & $(5,6)$ & 0,082 \\
\hline MG(kg) & 39,9 & (6.7) & 38,8 & $(10,4)$ & 0,809 \\
\hline$\% G T$ & 52,7 & $(4,7)^{a}$ & 46,0 & $(4,3)$ & 0,007 \\
\hline $\operatorname{MCM}(\mathbf{k g})$ & 38,8 & $(7,1)^{\mathrm{b}}$ & 45,3 & $(7,3)^{a b}$ & 0,085 \\
\hline $\mathrm{BMD}\left(\mathrm{g} / \mathrm{cm}^{2}\right)$ & 1,188 & $(0,06)^{a}$ & 1,123 & $(0,07)^{\mathrm{ab}}$ & 0,095 \\
\hline $\mathrm{BMC}\left(\mathrm{g} / \mathrm{cm}^{2}\right)$ & 2635,6 & $(427,5)^{b}$ & 2556,2 & $(327,5)^{\mathrm{ab}}$ & 0,661 \\
\hline
\end{tabular}

DP= Desvio Padrão; IMC=Índice de massa corpórea; \%GC=Gordura Corporal; MG= Massa Gorda; \%GT= Gordura de Tronco; $M C M=$ Massa Corporal Magra; $\mathrm{BMD}=$ Densidade mineral óssea; $\mathrm{BMC}=$ Conteúdo mineral ósseo; $\mathrm{p}<0,05=$ significância estatística; Letras minúsculas semelhantes $=$ Correlação entre variáveis. 
demostram que ha diferença estatística entre os gêneros apenas para o \% GT ( $\mathrm{p}=0,007)$. No grupo feminino houve correlação da BMD com o \%GC e o \%GT, já a BMC correlacionou-se com o peso e MCM. No grupo masculino houve correção da BMC e BMD com a estatura e MCM.

Após a intervenção, no grupo feminino, houve significância apenas na variável estatura $(\mathrm{p}=0,014)$, já no grupo masculino o aumento da estatura $(\mathrm{p}=0,0001), \mathrm{MCM}(\mathrm{p}=0,011)$ e $\mathrm{BMC}(\mathrm{p}=0,002)$ foi significantemente, bem como a diminuição dos valores de IMC ( $\mathrm{p}=0,004), \% \mathrm{GC}(\mathrm{p}=0,0001)$ e \%GT $(\mathrm{p}=0,016)$ (Tabela 2).

Após o treinamento 78,9\% dos adolescentes aumentaram e $21,1 \%$ diminuíram o BMD. Dos que aumentaram o BMD 63,2\% diminuíram o \%GC, $57,9 \%$ diminuíram a MG e 57,9 aumentaram a MCM (Tabela 3).

Tabela 2. Composição óssea e corporal pré e pós o treinamento concorrente.

\begin{tabular}{|c|c|c|c|c|c|c|c|c|}
\hline \multirow[b]{3}{*}{ Peso (kg) } & \multicolumn{4}{|c|}{ Feminino - Média (DP) } & \multicolumn{4}{|c|}{ Masculino - Média (DP) } \\
\hline & \multicolumn{2}{|c|}{ Pré } & \multicolumn{2}{|c|}{ Pós } & \multirow{2}{*}{$\begin{array}{c}\boldsymbol{P} \\
0,790\end{array}$} & \multirow{2}{*}{$\frac{\text { Pré }}{88,3(15,8)}$} & \multirow{2}{*}{$\begin{array}{c}\text { Pós } \\
88,1 \quad(14,6)\end{array}$} & \multirow{2}{*}{$\begin{array}{c}\boldsymbol{P} \\
0,862\end{array}$} \\
\hline & 83,8 & $(13,0)$ & 84,4 & $(15,5)$ & & & & \\
\hline Est (cm) & 158,7 & $(11,6)$ & 160,0 & $(11,7)$ & 0,014 & $165,9 \quad(6,6)$ & $168,8 \quad(6,3)$ & 0,0001 \\
\hline IMC & 33,1 & $(1,7)$ & 32,6 & $(2,0)$ & 0,547 & $32,0 \quad(5,7)$ & $30,9 \quad(5,0)$ & 0,004 \\
\hline$\% G C$ & 49,2 & $(4,6)$ & 48,9 & $(5,8)$ & 0,803 & $44,3 \quad(5,6)$ & $41,1 \quad(6,3)$ & 0,000 \\
\hline MG (kg) & 39,9 & $(6,7)$ & 40,6 & $(10,3)$ & 0,750 & $38,8 \quad(10,4)$ & $36,6 \quad(9,3)$ & 0,067 \\
\hline$\% G T$ & 52,7 & $(4,7)$ & 52,9 & $(6,2)$ & 0,893 & $46,0 \quad(4,3)$ & $43,3 \quad(5,9)$ & 0,016 \\
\hline MCM (kg) & 38,4 & $(7,9)$ & 38,8 & $(8,2)$ & 0,633 & $45,3 \quad(7,3)$ & $47,7 \quad(7,6)$ & 0,011 \\
\hline BMD $\left(\mathrm{g} / \mathrm{cm}^{2}\right)$ & 1,188 & $(0,06)$ & 1,196 & $(0,06)$ & 0,421 & $1,123(0,07)$ & $1,223(0,30)$ & 0,225 \\
\hline BMC (g/cm²) & 2635,6 & $(427,5)$ & 2736,0 & $(481,8)$ & 0,075 & $2556,2 \quad(327,5)$ & $2753,2 \quad(337,6)$ & 0,002 \\
\hline
\end{tabular}

DP= Desvio Padrão; IMC=Índice de massa corpórea; \%GC=Gordura Corporal; MG= Massa Gorda; \%GT= Gordura de Tronco; $\mathrm{MCM}=$ Massa Corporal Magra; $\mathrm{BMD}=$ Densidade mineral óssea; $\mathrm{BMC}=$ Conteúdo mineral ósseo; $\mathrm{p}<0,05=$ significância estatística .

Tabela 3. Frequência da densidade mineral óssea de adolescentes obesos de acordo com a composição corporal pós intervenção.

\begin{tabular}{|c|c|c|c|c|c|}
\hline & \multicolumn{5}{|c|}{ Densidade Mineral óssea } \\
\hline & & Diminuíram $(21,1 \%)$ & Aumentaram (78,9\%) & Total & $p$ \\
\hline \multirow[t]{2}{*}{$\%$ GC } & D & $21,1 \%$ & $63,2 \%$ & $84,2 \%$ & 1,000 \\
\hline & A & $0 \%$ & $15,8 \%$ & $15,8 \%$ & \\
\hline \multirow[t]{2}{*}{ MG (kg) } & D & $21,1 \%$ & $57,9 \%$ & $78,9 \%$ & 1,000 \\
\hline & A & $0 \%$ & $21,1 \%$ & $21,1 \%$ & \\
\hline \multirow[t]{2}{*}{ MCM (kg) } & A & $10,5 \%$ & $57,9 \%$ & $68,4 \%$ & 0,688 \\
\hline & D & $10,5 \%$ & $21,1 \%$ & $31,6 \%$ & \\
\hline
\end{tabular}

$\% G C=$ Gordura Corporal; $M G=$ Massa Gorda; $M C M=$ Massa Corporal Magra; $B M D=$ Densidade mineral óssea; $D=$ Diminuíram; $A=$ Aumentaram $p<0,05=$ significância estatística. 


\section{Discussão}

Estudos epidemiológicos revelam que crianças obesas apresentam grande probabilidade de serem adultos obesos e, consequentemente, iniciar o processo de desenvolvimento de diversas doenças crônicas de forma precoce ${ }^{14}$. Em contraste a estas consequências deletérias, a obesidade pode atuar como fator protetor a osteoporose e fraturas. ${ }^{15}$

Dimensões corporais e hormonais que conduzem o adolescente ao amadurecimento biológico são resultantes de vários elementos moduladores da aquisição da massa óssea, como aspectos genéticos, hormônios que agem no processo de calcificação, alterações no peso corporal, sobrecarga mecânica imposta ao esqueleto e ingestão adequada de vitamina ${ }^{16}$. A preservação da massa magra também é capaz de contribuir na manutenção da densidade mineral óssea por exercer um efeito osteogênico, provocando estresse mecânico e estímulos ao esqueleto (efeito piezoelétrico). ${ }^{17}$

De acordo com os resultados da presente pesquisa, no grupo feminino a BMD associou-se apenas a obesidade. Estudos demostram que a abundância de tecido adiposo dos obesos pode contribuir para o aumento da expressão de andrógenos em estrógenos, o que aumenta a concentração circulante de esteroides sexuais que, por sua vez, influenciariam no aumento da massa óssea, e que este aumento é superior em mulheres obesas, isto quando comparadas a mulheres eutróficas. ${ }^{18}$

No grupo masculino a associação com BMD ocorreu apenas com, estatura e massa magra. Já a BMC correlacionou-se com a massa magra em ambos os grupos, feminino e masculino. Estes resultados podem ter ocorrido em função de que a BMD atinge seu pico máximo durante a adolescência levando a uma aceleração da maturação esquelética, com o aumento da estatura e consequentemente da BMC. ${ }^{19}$

Como forma de prevenção e tratamento da obesidade e da massa óssea, existem diversos fármacos, no entanto, para adolescentes indica-se dieta balanceada, bem como a pratica de exercício físico. ${ }^{20}$

O treinamento concorrente agrega dois modelos de exercícios, resistido e aeróbio ${ }^{7,21}$, e mostra-se capaz de causar estímulos distintos ao corpo. Na presente pesquisa, o treinamento foi capaz de apresentar resultados significativos para estatura, tanto para o gênero feminino quanto masculino, isso em função de ser uma população em fase de crescimento e de- senvolvimento. ${ }^{22}$ Já o IMC e o percentual de gordura corporal, após o treinamento, diminuíram apenas nos meninos.

A redução do peso é associada, em adultos e idosos, à diminuição da BMD e do BMC, além de aumento importante no risco de fraturas com perda de peso superior a $10 \%$. Em adolescentes, os efeitos da obesidade e a perda de peso sobre a massa óssea não são totalmente elucidados e esse conhecimento adquire relevância, visto que o comprometimento do pico de aquisição de massa óssea e a incorporação de hábitos alimentares errôneos podem contribuir para o maior risco de baixa densidade óssea e de fraturas por fragilidades ósseas na vida adulta. ${ }^{23}$

No presente estudo, 21,1\% dos adolescentes diminuíram a densidade mineral óssea, destes, todos diminuíram também o \%GC, bem como a MG. Dos 78,9\% que aumentaram a BMD, 57,9\% também aumentaram a MCM. Como os jovens realizaram treinamento concorrente, acredita-se que a maioria tenha aumentado a BMD, em função do ganho de MCM.

A MCM aumentou significantemente apenas nos meninos, bem como a BMC. O aumento da massa magra, apenas no grupo masculino pode ter ocorrido devido a características do gênero ${ }^{24}$, já o BMC pode ter aumentado, em decorrência do aumento da MCM e consequentemente da BMD, tendo em vista que uma é consequência da outra, respectivamente. ${ }^{8}$

De acordo com os dados aqui apresentados, e com a literatura consultada, esta bastante clara a importância de se estudar o efeito do treinamento na densidade e o conteúdo mineral ósseo em adolescentes obesos, e a necessidade de analisar a amostra em um período mais longo para verificar possíveis resultados no grupo feminino, tento em vista que as mesmas diminuíram a gordura corporal, aumentaram a massa magra, BMC e BMD, mesmo não apresentando significância estatística. Entretanto, é necessário mencionar limitações no estudo, como a ausência do controle da dieta, amostra reduzida e ausência do grupo controle.

\section{Conclusão}

De acordo com os resultados conclui-se que as 16 semanas de treinamento concorrente foram efetivas para a diminuição da gordura corporal, aumento da massa magra e conteúdo mineral ósseo de adolescentes do gênero masculino. No grupo feminino não houve diferença do momento pré para o pós intervenção. 


\section{Referencias}

1. Lavrador MS, Abbes PT, Escrivão MA, Taddei JA. Cardiovascular risks in adolescents with different degrees of obesity. Arq Bras Cardiol. 2011;96:205-11.

2. Instituto Brasileiro de Geografia e Estatística (IBGE). POF 20082009 - Antropometria e estado nutricional de crianças, adolescentes e adultos no Brasil. 2010.

3. Wang YC, McPherson K, Marsh T, Gort-maker SL, Brown M. Health and eco-nomic burden of the projected obesity trends in the USA and the UK. Lancet. 2011;378(9793):815-25.

4. Gracia-Marco L, Ortega FB, Jimenez-Pavon D, Rodriguez G,Castillo MJ, Vicente-Rodriguez G, Moreno LA (2011) Adiposity and bone health in Spanish adolescents. The HELENA study. Osteoporos Int 23:937-47.

5. Azevedo FR, Brito BC. Influência das variáveis nutricionais e da obesidade sobre a saúde e o metabolismo. Rev Assoc Med Bras. 2012;58:714-23.

6. Fagherazzi S, Dias RL, Bortolon F. Impacto do exercício físico isolado e combinado com dieta sobre níveis séricos de HDL, LDL, Colesterol total e triglicerídeos. Rev Bras Med Esporte. 2008;14: 381-6.

7. Davis JN, Tung AMY, Chak SS, Ventura EE, Byrd-Williams $\mathrm{CE}$, Alexander KE et al.. Aerobic and Strength Training Reduces Adiposity in Overweight Latina Adolescents. Med Sci Sports Exerc. 2009;41:1494-503.

8. Cadore EL, Brentano MA,Kruel LFM. Efeitos da atividade física na densidade mineral óssea e na remodelação do tecido ósseo. Rev Bras Med Esporte. 2005;11: 373-9.

9. Cole TJ, Belizzi MC, Flegal KM, Dietz WH. Establishing a standard definition for child overweight and obesity worldwide: international survey. Br Med. J. 2000; 320:1240-3.

10. Freitas Júnior IF, et al. Padronização de técnica antropométricas. São Paulo: Cultura Acadêmica, 2008.

11. Faigenbaum AD, Westcott WL. Youth strength training: programs for health. Fitness and sport. 2ed. Champaign. Human Kinetics, 2009.

12. Bentley DJ, Newell J, Bishop D. Incremental exercise test design and analysis implications for performance diagnostics in endurance athletes. Sports Med. 2007; 37: 575-86

13. Baechle TR, Groves BR. Weigth Training. Champaign: Leissure Press, 1992.

14. Junqueira CLC, Costa GM, Magalhães MEC. Síndrome Metabólica: O risco cardiovascular é maior que o risco dos seus componentes isoladamente? Rev Bras Cardiol. 2011;24: 30815.
15. Rocher E, Chappard C, Jaffre C, Benhamou L, Courteix D. Bone mineral density in prepubertal obese and control children: relation to body weight, lean mass, and fat mass. J Bone Miner Metab. 2008;26:73-8.

16. Rodrigues AM, Cintra IP, Santos LC, Martini LA, Mello MT, Fisberg M . Densidade mineral óssea, composição corporal e ingestão alimentar de adolescentes modelos de passarela. J Pediatr.2009;85:503-8.

17. Wang MC, Bachrach LK, Van Loan M, Hudes M, Flegal KM, Crawford PB. The relative contributions of lean tissue mass and fat mass to bone density in young women. Bone. 2005;37:474-81.

18. Hadji P, Bock K, Gotschalk M, Hars O, Backus J, Emons G, et al. The influence of serum leptin concentration on bone mass assessed by quantitative ultrasonometry in pre and postmenopausal women. Maturitas. 2003;44:141-8.

19. Bandeira F. A Obesidade Realmente Fortalece os Ossos? Arq Bras Endocrinol Metab. 2007;51: 895-7.

20. Reuter C, Stein CE, Vargas DM. Massa óssea e composição corporal em estudantes universitários. Rev Assoc Med Bras. [online]. 2012;58: 328-34.

21. Donnelly JE, Blair SN, Jakicic JM, Manore MM, Rankin JW, Smith BK. American College of Sports Medicine Position Stand. Appropriate physical activity intervention strategies for weight loss and prevention of weight regain for adults. Med Sci Sports Exerc. 2009;41:459-71.

22. Silva CC, Goldberg TBL, Teixeira AS, Dalmas JC. Análise Preditiva da Densidade Mineral Óssea em Adolescentes Brasileiros Eutróficos do Sexo Masculino. Arq Bras Endocrinol Metab. 2006;50:105-13.

23. Santos LC, Cintra IP, Fisberg M, Castro ML, Martini LA. Associação entre a Perda de Peso, a Massa Óssea, a Composição Corporal e o Consumo Alimentar de Adolescentes Obesos Pós-Púberes. Arq Bras Endocrinol Metab. 2008;52:1001-8.

24. Prado WL, Siegfried A, Dâmaso AR, Carnier J, Piano A, Siegfried W. Efeitos da terapia multidisciplinar de longo prazo sobre a composição corporal de adolescentes internados com obesidade severa. J Pediatr. (Rio J.) [online]. 2009;85:243-8. 